Apidologie, 1980, 11 (4), 375-383.

\title{
DER HYDROXYMETHYLFURFURALGEHALT IN HONIGEN DER BUNDESREPUBLIK DEUTSCHLAND AUSWERTUNG DER MESSUNGEN IN DEN JAHREN 1970-1979
}

\section{La teneur en hydroxyméthylfurfural des miels de la République fédérale d'Allemagne Explottation des mesures fattes de 1970-1979}

\author{
Günther VORWOHL \\ Landesanstalt für Bienenkunde Universität Hohenheim \\ August von Hartmann Str. 13, D 7000 Stuttgart 70, Bundesrepublik Deutschland
}

\section{SUMMARY}

THE HYDROXYMETHYLFURFURAL CONTENT IN HONEYS OF THE FEDERAL REPUBLIC OF GERMANY

DATA FROM DETERMINATIONS BETWEEN 1970 AND 1979

The average HMF content of 7919 samples was $4,1 \mathrm{ppm}(=0,41 \mathrm{mg} \%)$. The range of values is given in tables. Domestic honeys show low HMF values in comparision with data published in the international literature.

\section{ZUSAMMENFASSUNG}

Der durchschnittliche HMF-Gehalt in 7919 Honigen belief sich auf $4,1 \mathrm{ppm}(=0,41 \mathrm{mg} \%)$. Die Streuung der Werte wird in Tabellen dargestellt. Die einheimischen Honige zeigen im Vergleich mit den Zahlen, die in der internationalen Literatur veröffentlicht wurden, niedrige HMF-Gehalte.

\section{EINLEITUNG}

Der Gehalt an Hydroxymethylfurfural (im folgenden HMF genannt), dient als Gradmesser für die Wärmeeinwirkungen, denen ein Honig während der Gewinnung, Klärung, Lagerung, Abfüllung und Vermarktung unterworfen war. Bei HMFGehalten von mehr als $200 \mathrm{ppm}(=20 \mathrm{mg} \%)$ ist auch die Frage zu prüfen, ob eine Verfälschung mit künstlich hergestelltem Invertzucker vorliegt.

Die Auswertung grosser Messreihen kann dazu helfen, vernünftige Höchstwerte festzulegen und zu prüfen, ob die bestehenden höchstzulässigen Werte einerseits die Erfordernisse der Praxis berücksichtigen, die auf Wärmeanwendungen und Lagerhaltung nicht verzichten kann und andererseits Gewähr dafür leisten, dass der Honig so " naturbelassen " wie möglich den Verbraucher erreicht. Die Verordnung über 
Honig der Bundesrepublik Deutschland legt für Speisehonig als höchstzulässigen Wert $40 \mathrm{ppm}$ (=4 mg \%) HMF fest. Mit anderen Worten, ein Kilogramm Honig darf höchstens vierzig Milligramm HMF enthalten, oder was auf das gleiche hinausläuft, hundert Gramm Honig höchstens vier Milligram HMF. Der gleiche Wert steht in der Richtlinie der Europäischen Gemeinschaft und im Recommended European Standard for Honey der FAO/WHO. Der Deutsche Imkerbund hat für Honig unter seinem Markenzeichen $15 \mathrm{ppm}(=1,5 \mathrm{mg} \%)$ als höchstzulässigen Wert festgelegt. Dieser Regelung hat sich die C.M.A. (Centrale Marketinggesellschaft der deutschen Agrarwirtschaft) und die Landwirtschaftskammer Schleswig Holstein für ihre Warenzeichen angeschlossen.

\section{MATERIAL UND METHODEN}

Ausgewertet wurden 7919 HMF-Messungen an Honigen aus der gesamten Bundesrepublik. Es wurden nur Honige berücksichtigt, bei denen die mikroskopische Untersuchung die einheimische Herkunft bestätigte. Die Messung erfolgte nach dem colorimetrischen Verfahren von WINKLER (1955). Dabei wurden die bei VoRwoHL (1969) näher beschriebener Kautelen beachtet. Für die Messungen stand bis 1976 ein Zeiss Colorimeter, Typ Elko II, zur Verfügung, ab 1976 ein Spektralphotometer der gleichen Firma der Type PM 2 DL.

\section{ERGEBNISSE}

Im Durchschnitt aller 7919 Messungen ergab sich ein HMF-Gehalt von 4,1 ppm. Das ist etwas mehr als der zehnte Teil der höchstzulässigen Menge des Recommended European Standard, der EG-Richtlinie und der dieser folgenden nationalen Verordnungen und weniger als ein Drittel der nach den DIB-Richtlinien statthaften Menge.

Das Probenmaterial lässt sich in die folgenden Kategorien aufteilen :

\section{a) Ausstellungshonige}

Die Deutsche Landwirtschaftsgesellschaft und die regionalen Landesverbände der Imkerschaft veranstalten von Zeit zu Zeit Honigprämiierungen. Diese Wettbewerbe werden der Natur der Sache nach von geschulten Imkern beschickt.

Entsprechend niedrig liegt der durchschnittliche HMF-Gehalt, nämlich bei 2,7 ppm. Die Kategorie Ausstellungshonige umfasst 2222 Proben. Es handelt sich um verkaufsfertig abgefüllte Gläser.

\section{b) CMA-Kontrollen}

Nur geringfügig höher liegt der durchschnittliche HMF-Gehalt in der Kategorie der sogenannten CMA-Kontrollen. Dabei handelt es sich um Honigproben, die auf Anforderung der Centralen Marketinggesellschaft der deutschen Agrarwirtschaft direkt bei den Imkern und lizensierten Abfüllern erhoben werden. Die Proben stammen also aus der laufenden Produktion und den Lagerbeständen. In diese Kategorie fallen 1270 Proben, der durchschnittliche HMF-Gehalt liegt bei $2,9 \mathrm{ppm}$. 


\section{c) Voruntersuchungen}

In der Kategorie Voruntersuchungen wurden Honige vereinigt, die unaufgefordert, entweder von Imkern oder Abfüllern eingesandt wurden, um Auskunft über Herkunft und Qualität des Produktes zu erhalten. Bei 3246 Voruntersuchungen ergab sich ein durchschnittlicher HMF-Gehalt von 4,4 ppm. Als Voruntersuchungen kommen unter anderen Proben ins Labor, die älteren Lagerbeständen entstammen und daher überprüft werden sollen, ob sie noch verkehrsfähig sind. Ferner fallen in dieser Gruppe auch Proben von Honigen, die zur Verflüssigung erwärmt wurden und bei denen eventuelle Wärmeschäden quantifiziert werden sollen. Proben der geschilderten Art treiben den Durchschnittswert nach oben.

\section{d) Marktkontrollen}

Unter Marktkontrollen verstehen wir Honige unter dem Markenzeichen des DIB, die in Ladengeschäften und bei Kontrollen lizensierter Abfüller erhoben werden. 1181 Honige fallen in diese Kategorie. Der durchschnittliche HMF-Gehalt liegt bei 8,1 ppm. Bei einem zulässigen Höchnstwert von 15 ppm ist dies immer noch ein günstiges Ergebnis, die Differenz zu den anderen Kategorien jedoch beachtlich. Zur Erklärung können zwei Tatbestände herangezogen werden. Der Detailhandel wird insbesondere von lizensierten Abfüllstellen beliefert, die Honig im grösseren Massstab abfüllen. Die Abfüllung im grossen Stil führt zwangsläufig zu grösseren Wärmebelastungen als sie im Kleinbetrieb notwendig sind. Bekanntlich liegt der durchschnittliche Völkerbestand der Imker der Bundesrepublik Deutschland bei zehn Völkern. Damit bleibt auch die Menge des im Laufe des Jahres zu bearbeitenden Honigs unter einer Tonne. Die Honige aus den Kleinbetrieben werden überwiegend direkt an den Verbraucher geliefert und werden bei Marktkontrollen nur in geringem Umfang erfasst. Ferner herrscht bei Marktkontrollen die Tendenz zu negativer Auswahl. Es werden im Detailhandel bevorzugt Proben aufgegriffen, die schon äusserliche Mängel aufweisen, etwa unschöne Kandierung, die unter Umständen durch lange Lagerung oder übermässige Erwärmung bedingt ist.

Neben den durchschnittlichen Werten interessiert natürlich auch die Streuung des HMF-Gehalts. Dazu wurde eine Klassifizierung vorgenommen. Die Klasse I umfasst alle Proben mit 0-5 ppm HMF, die Klasse II alle Proben mit 5,1 bis $10 \mathrm{ppm}$ und so fort.

Ab Klasse $\mathrm{V}$ beträgt der Klassenabstand $10 \mathrm{ppm}$. Die Klasse $\mathrm{V}$ enthält also alle Proben mit HMF-Gehalten von $20,1 \mathrm{ppm}$ bis $30 \mathrm{ppm}$. In die Klasse VIII fallen alle Proben zwischen 50 und 100 ppm HMF und in die Klasse IX alle Honige mit HMFGehalten über $100 \mathrm{ppm}$ HMF.

Alle Honige, die hinsichtlich des HMF-Gehalts den Richtlinien des DIB entsprechen, fallen demnach in die Klassen I-III. Die Klasse IV umfasst die Proben, die den höchstzulässigen Wert geringfügig überschreiten. Die Proben in den Klassen IV-VI genügen noch den Anforderungen der Honigverordnung der Bundesrepublik Deutschland, der EG-Richtlinie und dem Recommended European Standard für Speisehonig. Die Klasse VII umfasst wiederum die Honige, die den Grenzwert 40 ppm geringfügig 
überschreiten. Die Honige über $10 \mathrm{ppm}$ sind nur als Back- oder Industrie-Honige verkehrsfähig. In der Tabelle 1 sind die Ergebnisse der HMF-Messung an Ausstellungshonigen, Marktkontrollen usw. nach Klassen aufgeschlüsselt.

Tabelle 2 fasst die Untersuchungsergebnisse nach Jahren zusammen, wobei die Honigproben aller Kategorien zusammen addiert wurden. Das beste Ergebnis findet sich im Jahre 1976, das als ausserordentlich gutes Honigjahr in Erinnerung ist. Das bedingt einen hohen Prozentsatz von Honigen neuer Ernte in den Proben aller Kategorien. Nach 1976 nimmt die Anzahl der Honige in der Klasse I wieder etwas ab. Die Jahre 1977-1979 brachten auf die gesamte Bundesrepublik bezogen nur mässige Ernten. Man darf also annehmen, dass Lagerbestände insbesondere von 1976 mobilisiert wurden, was sich bei den HMF-Werten niederschlägt.

Verfolgt man die Prozentsätze der Proben, deren HMF-Wert in die Klasse I fällt im Laufe der Jahre, so zeigt sich eine leicht positive Tendenz. Diese ergibt sich auch, wenn man die Auswertung der Jahre 1967, 1968 und des ersten Halbjahres 1969 mit einbezieht (VoRwOHL, 1969). In diesem Zeitraum fielen $74,8 \%$ der Proben in die Klasse I. Seit 1974 sind in dieser Klasse keine Prozentsätze unter $80 \% \mathrm{mehr}$ vorgekommen.

Dies kann als Erfolg der kontinuierlichen Kontrollen und der zusätzlichen Aufklärung durch Wort und Schrift gewertet werden.

\section{DISKUSSION}

WHITE veröffentlichte 1980 eine Literaturübersicht über die HMF-Gehalte in Honigen im internationalen Handel, wobei vorwiegend Honige vor der Abpackung berücksichtigt wurden. Der durchschnittliche Gehalt liegt bei 12,4 ppm.

Der HMF-Gehalt von 481 Honigen von Imkern in den Vereinigten Staaten lag im Durchschnitt bei $6,2 \mathrm{ppm}$ und variierte zwischen 0 und $136 \mathrm{ppm}$. 41 Proben von Wabenhonig enthielten zwischen 0,3 und 9,2 ppm HMF, bei einem durchschnittlichen Gehalt von $2,7 \mathrm{ppm}$. Die Verteilung der Werte ähnelt der für Honige der Bundesrepublik. Die überwiegende Mehrheit der Honige fällt in die Klasse I (0-5 ppm) (WhITE und Siciliano, 1980).

Nach den gleichen Autoren führt die Abfüllung in Gläser, Kannen oder Tonnen bei 7 grossen Abfüllern der Vereinigten Staaten zu einem durchschnittlichen Anstieg um 15,8 ppm HMF.

CURYLo (1972) gibt für 110 polnische Honigproben Werte zwischen 2,7 und $5 \mathrm{ppm}$ an.

In einer Untersuchung über 121 Marktkontrollen, die in allen Teilen Italiens erhoben worden waren, stellten D'AMBRosio und RICCIARDELLI D'ALBORE (1977) fest, dass nur 28 Muster HMF-Werte zwischen 0 und 15 ppm zeigten; 47 Honige enthielten 


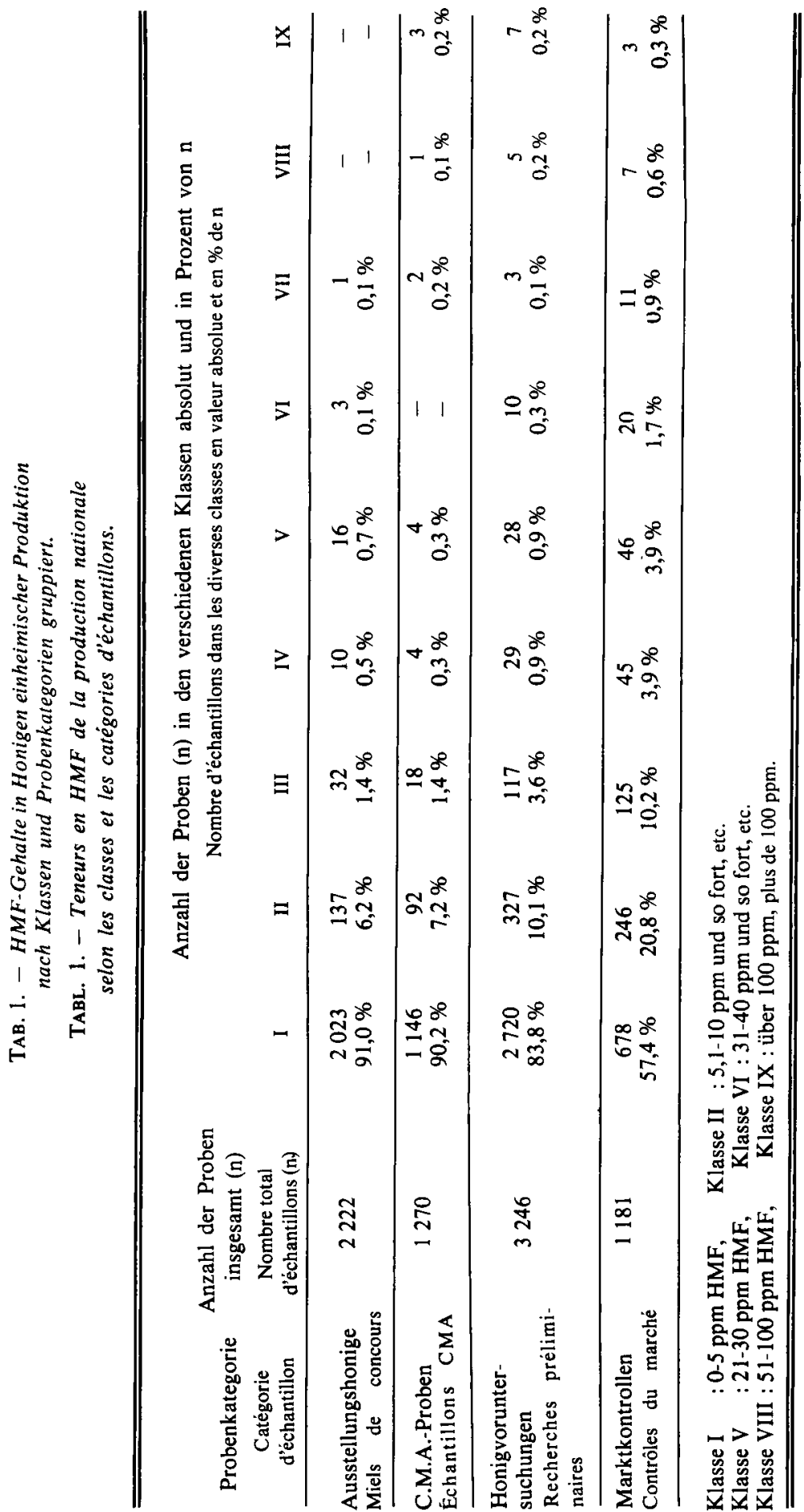


G. VORWOHL

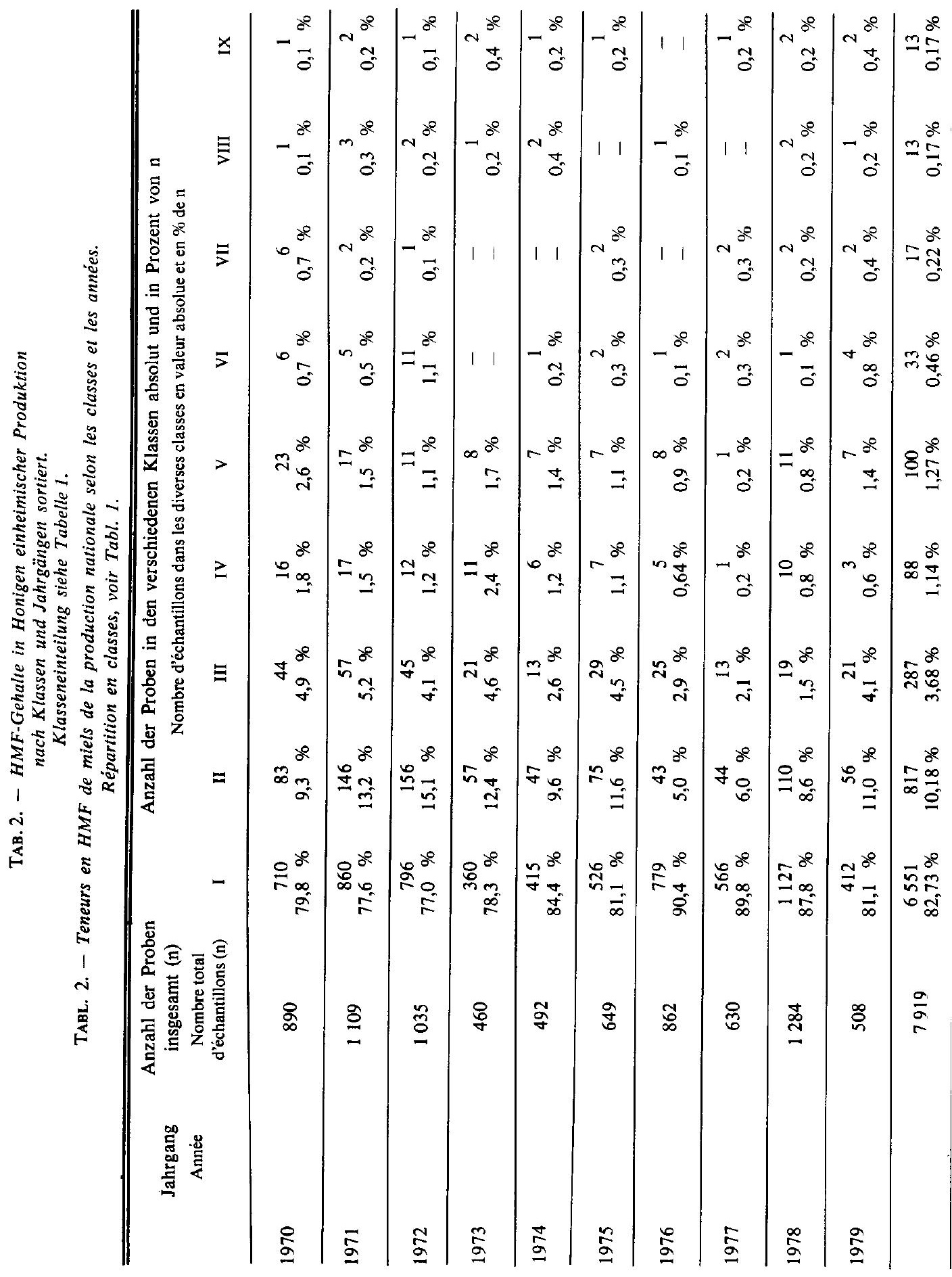


zwischen 15,1 und $40 \mathrm{ppm} ; 33$ Proben fielen in den Bereich zwischen 40,1 und 200 ppm und 13 der aufgekauften Produkte brachte es auf mehr als 200 ppm HMF. Durchschnittlich ergab sich ein HMF-Gehalt von 224,7 ppm, ein ungewöhnlich hoher Wert, der hauptsächlich durch 13 extreme HMF-Gehalte von über $1000 \mathrm{ppm}$ bedingt wird. Die Auswertung zeigt nachdrücklich wie sich das jahrelange Fehlen einer effizienten Kontrolle auf die Qualitätsverhältnisse auf einem nationalen Markt auswirkt. Das untersuchte Material umfasste einheimische und nach Italien importierte Honige.

FiNI und SABATINI (1972) untersuchten 800 italienische Honige, von denen 400 direkt von Imkern, Imkervereinigungen und Genossenschaften bezogen wurden und 400 Honige aus dem Einzelhandel. Die Proben, die beim Erzeuger erhoben worden waren, zeigten einen durchschnittlichen HMF-Gehalt von 13,4 ppm. 365 der Muster fielen in die erste Klasse (0-10 ppm). Die Honige aus dem Handel enthielten im Durchschnitt 59,6 ppm HMF. Nur 135 Proben hatten HMF-Gehalte zwischen 0 und $10 \mathrm{ppm}$.

Nach Gassparuan und VorwoHL (1974) lagen die HMF-Gehalte von 100 Honigproben, die im Iran, insbesondere in der Region Isfahan aufgekauft wurden, in über 45 Fällen über $40 \mathrm{ppm}$ und nur in 37 Fällen unter $15 \mathrm{ppm}$. Die Verhältnisse gleichen denen auf dem italienischen Markt.

HALLERMAYER gab 1969 den durchschnittlichen HMF-Gehalt von 1500 nicht näher beschriebenen Importhonigen mit $33 \mathrm{ppm}$ an. Es handelte sich offensichtlich um Honige, die weder sorgfältig gelagert noch mit der nötigen Schonung abgefüllt worden waren.

Über den HMF-Gehalt australischer, für den Export bestimmter Honige berichtete LaNGridge (1977). Von den 186 getesteten Proben fallen $92 \%$ in die Klassen mit 0$15 \mathrm{ppm}$ HMF. Die Klassen mit mehr als $40 \mathrm{ppm}$ sind nur minimal besetzt.

Ricciardelli D'Albore (1978) fand in 30 Honigen aus dem Staate Santa Catarina, Brasilien, einen durchschnittlichen HMF-Gehalt von $47,5 \mathrm{ppm}$. Als niedrigster Wert wurden $2,5 \mathrm{ppm}$ gemessen und als höchster $346,1 \mathrm{ppm}$. 10 Honige zeigten Werte zwischen 0 und 15 ppm, 15 Proben fielen in den Bereich zwischen 15,1 und $40 \mathrm{ppm}$ und 5 weitere Muster lagen - meist erheblich - über $40 \mathrm{ppm}$.

VoRwOHL (1976) publizierte die Ergebnisse von 42 HMF-Messungen an Honigen aus dem tropischen Afrika. 12 Proben enthielten HMF in der Grössenordnung von 0$5 \mathrm{ppm}$, weitere 12 zeigten Werte zwischen 5,1 und $15 \mathrm{ppm}, 10$ Messwerte fielen in den Bereich zwischen 15,1 und $40 \mathrm{ppm}$ und 8 über $40 \mathrm{ppm}$.

ZüRCHER u.a. untersuchten 197554 Honige aus dem Detailhandel in der Schweiz, die teils einheimisch teils aus einer grösseren Anzahl von Ländern importiert worden waren. 11 Proben enthielten 0-5 ppm HMF, bei 21 Mustern ergaben sich Werte zwischen 5,1 und 15 ppm, 18 Honige zeigten HMF-Gehalte zwischen 15,1 und 40 ppm, 4 Proben fielen in die Kategorie der Backhonige mit mehr als 40 ppm. 
Die HMF-Gehalte der Honige der Bundesrepublik Deutschland liegen also im Vergleich mit den bisher veröffentlichten Werten der internationalen Literatur sehr niedrig.

Die derzeitig gültigen höchstzulässigen Werte für den HMF-Gehalt des Honigs sind offensichtlich praktikabel, allenfalls wäre an eine Herabsetzung zu denken.

\section{DANK}

Frau H. MAGENAU danke ich für die wenig unterhaltsame Zusammenstellung der HMF-Werte aus vielen Tausenden von Untersuchungsprotokollen und für die mit der Auswertung verbundene Rechenarbeit.

\section{RÉSUME}

La teneur en hydroxymethylfurfural (HMF) sert à mesurer l'action de la chaleur à laquelle un miel a été soumis durant la récolte, la décantation, le stockage, le conditionnement et les traitements divers.

La mesure a été faite selon la méthode de WiNKLER (1955) à l'aide d'un photomètre Zeiss Elko II et (à partir de 1976) d'un spectophotomètre PM 2 DL de la même firme.

7919 miels produits en République fédérale d'Allemagne ont été étudiés. L'exactitude de la mention d'origine a èté vérifiée au microscope.

La teneur moyenne en HMF se situe autour de $4,1 \mathrm{ppm}(=0,41 \mathrm{mg} \%)$.

Les miels envoyés par les apiculteurs pour être primés ont les teneurs les plus basses (2,7 ppm). Des teneurs en HMF légèrement plus èlevées n'ont été trouvées que pour des miels provenant de stocks d'apiculteurs et de conditionneurs licenciés, fournis par la CMA (Société centrale de marketing de l'agriculture allemand) (2,9 ppm HMF).

Dans la catégorie "recherches préliminaires ", dont les miels ont été envoyés par des apiculteurs ou des conditionneurs pour tester leur qualité, la teneur moyenne en HMF est de 4,4 ppm.

On trouve en moyenne 8,1 ppm d'HMF dans les miels qui ont été fournis par les contrôles du marché, donc dans le commerce de détail, ou par les contrôles des conditionneurs licenciés. Le tableau 1 donne la dispersion des teneurs en HMF.

Au cours des douze dernières années, les teneurs en HMF ont montré une légère tendance à la baisse (Tabl. 2). Les résultats les meilleurs ont été enregistrés pour l'année 1976, qui s'est caractérisée par une récolte de miel particulièrement importante. Dans les années 1977-1979, qui ont fourni des récoltes médiocres, le résultat s'est altéré, en raison de la mobilisation des stocks, dont les miels renferment en général plus d'HMF que les miels fraîchement récoltés.

La norme européenne FAO/WHO recommandée pour le miel, la directive de la Communauté européenne et le décret national de la RFA limitent la teneur en HMF des miels de bouche à $40 \mathrm{ppm}$. Le Deutscher Imkerbund ne permet que $15 \mathrm{ppm}$ pour les miels sous son label. Le pourcentage des miels indigènes, qui ne satisfont plus à ces directives, est faible. C'est pourquoi, par rapport aux valeurs internationales, les teneurs en HMF des miels de la RFA sont faibles.

\section{LITERATUR}

CURYLo J., 1972. - Content of 5-hydroxymethylfurfural in Polish honey. Pszeln. zesz. Nauk., 16, 147-151 (in polnisch).

D'Ambrosio Marina, Ricciardelli D'Albore G. C., 1976. - Ulteriori osservazioni sui mieli commerciati in Italia. Ann. Istituto Sperm. Zool. Agrar., 5, 15-20. 
Duisberg H., Hadorn H., 1966. - Welche Anforderungen sind an Handelshonige zu stellen? Mittl. Lebensmittelunters. Hygiene, 57 (5), 386-407.

FinI Maria Angela, SABATINI Anna Gloria, 1972. - Indagine Comperativa sul contenuto in idrossimetilfurfuralo dei mieli. S.e.TA., 2 (6), 375-379.

GASSPARIAN S., VoRwOHL G., 1974. - Vergleichende Qualitätsuntersuchungen an iranischen Honigen. Apidologie, 5 (2), 177-190.

HALleRmayer R., 1969. - Beitrag zur Beurteilung von Bienenhonig. Gordian, (5), 230-234.

LANGRIDGE D. F., 1977. - Australian honey on the international market. Symposio internationale di technologia apistica. Bologna 1977. Apimondia Verlag Bukarest, pp. 46-54.

RuCCIARDelli D'AlBore G. C., 1978. - L'apicoltura nello stato di S. Catarina (Brasile) : Caratteristiche microscopiche e fisico-chimiche dei mieli che vi si producono. Riv. Agricolt. Subtropic. Tropic., 72 (3-4), 271-290.

VoRwoHL G., 1969. - Der Hydroxymethylfurfuralgehalt in deutschen Honigen. Z. Bienenforsch., 9 (11/12), 504-508.

VoRwOHL G., 1976. - Honeys from tropical Africa : microscopical analysis and quality problems. Apiculture in tropical climates, Ed. E. Crane, International Bee Research Association, London, 93-101.

WHrTE J. W., 1980. - Hydroxymethylfurfural content of honey as an indicator of its adulteration with invertsugar. Bee World, 61 (1).

Whrte J. W., Siciliano J., 1980. - Hydroxymethylfurfural and honey adulteration. J. Assoc. Off. Anal. Chem., 63 (1), 7-10.

WINKLER O., 1955. - Beitrag zum Nachweis und zur Bestimmung von Oxymethylfurfural. Z. Lebensmittel Unters. Forsch., 102, 3, 161-167.

ZürCher K., MAURIZIo Anna, HAdoRN H., 1975. - Untersuchungen an Handelshonigen mit spezieller Berücksichtigung des Zuckerspektrums. Apidologie, 6 (1), 59-90. 